\title{
Correlated Soft X-ray and Cryogenic Fluorescence Tomography Is a Powerful Tool to Explore the Role of Mitochondria-associated Membranes in Insulin Secretory Pathway
}

Valentina Loconte ${ }^{1}$, Jian-Hua Chen ${ }^{1}$, Axel Ekman ${ }^{1}$, Mark A. Le Gros ${ }^{2}$, Gerry Mc Dermott ${ }^{1}$, Kate White ${ }^{3}$ and Carolyn Larabell ${ }^{2}$

${ }^{1}$ Lawrence Berkeley National Laboratory and UCSF, Berkeley, California, United States, ${ }^{2}$ Molecular Biophysics and Integrated Bioimaging Division, Lawrence Berkeley National Laboratory, Berkeley, California, United States, ${ }^{3}$ University of Southern California, Los Angeles, California, United States

One of the key features of the insulin granule secretory pathway is their response to the variation of glucose concentration in the blood after the meal. It is reported that glucose-evoked insulin secretion exhibits a biphasic mechanism which is associated with the movement and acceleration of the granules in the cell, promoting the translocation from the perinuclear area toward the plasma membrane (Dehghany et al., 2015; Suckale \& Solimena, 2010). The mechanism that explains the relationship between these two events seems to be related to the role of mitochondria. In fact, our preliminary observations report a tight connection between granules and mitochondria, due to their spatial vicinity and non-random distribution of the granules with respect to mitochondria. These observations pinpoint the possible role of mitochondria as booster in granule kinetics (Dehghany et al., 2015); whether this mechanism may influence a specific stage of the granule maturation process is part of our investigation. The role of mitochondria in the insulin secretion involves also the possible change of granule composition, which may depend on the enhancement of the fatty acids (MacDonald et al., 2015) production and may involve other relevant compartments, such as ER. In order to explore the organelle localization in the cell, we used soft $\mathrm{X}$-ray tomography, a technique that visualizes and quantifies cell components in the 3D space, based on the absorption of the different chemical composition of each sub-cellular compartment. The absorption of the beam depends on the chemical composition and the thickness of each subcellular compartment (Le Gros et al., 2014). However, this may become a limitation when many organelles absorb with the same absorption coefficient. For example, it is already verified that under soft X-ray beam, ER, Golgi and cytoskeleton in beta cells are indistinguishable. In order to specifically localize subcellular compartments indistinguishable to SXT that are involved in the interaction of both insulin secretory granules and mitochondria, and to identify their influence on the spatial organization of the cell, correlated cryogenic fluorescence tomography (CFT) was used (Smith et al., 2014; Smith et al., 2012). In correlative CFT-SXT imaging, the cryopreserved specimen is imaged in a cryogenic high-numerical aperture light microscope prior to being transferred for acquisition of SXT data. In such analyses, labeled molecules are visualized precisely within high-resolution reconstructions of the cell, by colocalization of interacting organelles. An example is given by the ER, the compartment where proinsulin is folded (Suckale and Solimena, 2010). It is known to be in tight contact with mitochondria through Mitochondrial-Associated Membranes (MAMs) (Vance, 2014); therefore, unveiling the ER-mitochondria relationship will identify a direct (or indirect) cause for the granule spatial distribution. In summary, ER, Golgi, mitochondria, and lysosomes have been labelled by organic dyes and their positions recorded in relationship to the relative location of insulin granules.

\section{References}

- Dehghany J. et al. A Spatial Model of Insulin-Granule Dynamics in Pancreatic Beta-Cells, Traffic 16, 797-813 (2015). 
- Suckale, J. and Solimena, M. The insulin secretory granule as a signaling hub. Trends Endocrinol Metab 21, 599-609 (2010).

- MacDonald, MJ., et al., Characterization of Phospholipids in Insulin Secretory Granules and Mitochondria in Pancreatic Beta Cells and Their Changes with Glucose Stimulation. J Biol CHem 290(17): 11075-11092 (2015).

- Le Gros, M. A. et al. Biological soft X-ray tomography on beamline 2.1 at the Advanced Light Source. Journal of synchrotron radiation 21, 1370-1377, doi:10.1107/S1600577514015033 (2014).

- McDermott, G. et al. Visualizing cell architecture and molecular location using soft x-ray tomography and correlated cryo-light microscopy. Annu Rev Phys Chem 63, 225-239, doi:10.1146/annurevphyschem032511-143818 (2012).

- Smith, E. A. et al. Quantitatively imaging chromosomes by correlated cryo-fluorescence and soft xray tomographies. Biophys J 107, 1988-1996, doi:10.1016/j.bpj.2014.09.011 (2014).

- Vance, J. E., MAM(Mitochondria-Associated Membrans) in Mammalian cells: Lipids and Beyond. Biochim et Biophys Acta 1841, 595-609 (2014). 